\title{
Dynamic Monte Carlo with a proper energy barrier: Surface diffusion and two-dimensional domain ordering
}

\author{
H. C. Kang and W. H. Weinberg \\ Division of Chemistry and Chemical Engineering, California Institute of Technology, Pasadena, California \\ 91125
}

(Received 1 August 1988; accepted 18 November 1988)

\begin{abstract}
A new model is presented and discussed that allows Monte Carlo simulations to be carried out with a proper energy barrier crossing. Results are presented for the surface diffusion coefficient and the growth exponent of domain ordering of a half-monolayer of adatoms experiencing nearest- and next-nearest-neighbor repulsive lateral interactions (equal in magnitude), both on a square lattice. The results are compared with those derived using both Kawasaki dynamics and a Metropolis walk. The reasons why neither of the latter methods can be expected, in general, to describe thermally excited, time-dependent phenomena are explained and discussed.
\end{abstract}

\section{INTRODUCTION}

Monte Carlo simulations are widely used ${ }^{1-3}$ to study both the dynamic and the static behavior of systems with many interacting particles. In the investigation of static behavior, the simulations can be used to generate the statistically most important configurations. One commonly used algorithm is the Metropolis walk, ${ }^{4}$ which samples the configurations as follows. From an initial configuration, a new configuration can be generated, for instance, by a single spin flip in an Ising model. The new configuration is accepted with probability unity if its energy is less than that of the original configuration. Otherwise, it is accepted with probability $e^{-\left(E_{f}-E_{i}\right) / k_{B} T}$, where $E_{f}$ and $E_{i}$ are the energies of the new and the old configurations, respectively. Such algorithms search for the configurations of lowest free energy. For studying static behavior, the sequence of configurations that are generated need not correspond to the real evolution of the system towards the state of lowest free energy. The problem of conversion from simulated time $t_{s}$ to real time $t_{r}$ does not arise.

In studying dynamic behavior, however, the sequence of configurations generated by the Monte Carlo procedures is meant to simulate the time evolution of the system, and, hence, it is important to know how to convert $t_{s}$ to $t_{r}$ in this case. (Most Monte Carlo studies have reported their results in terms of $t_{s}$ ) At the very least, it is necessary to know that $t_{s}$ scales linearly with $t_{r}$. Investigating the dynamic behavior of a system by Monte Carlo techniques is equivalent to approximating the actual behavior of the system by a stochastic process. ${ }^{1,2}$ Consider the motion of adsorbed molecules on a crystal surface. This is often simulated by a lattice gas, the particles of which migrate by hopping from one site on the lattice to another. Frequently, the lattice-gas particles are only allowed to hop from their original sites to vacant, nearest-neighbor sites. The lattice-coordination number $z$ is dictated by the symmetry of the crystal surface, and the latticegas particles are allowed to interact with each other. A lattice-gas particle is picked randomly from an initial configuration. Then one of the $z$ nearest-neighbor sites is picked randomly. If the chosen nearest-neighbor site is occupied, the procedure is begun again. If the chosen nearest-neighbor site is vacant, the probability $w\left(c_{i}, c_{f}\right)$ of a successful hop from the original site to the chosen site is computed and compared to a random number $r(0 \leqslant r \leqslant 1)$. The particle then hops if $w\left(c_{i}, c_{f}\right) \geqslant r$, otherwise, it remains at its original site. The configuration is changed from $c_{i}$ to $c_{f}$ because of the hop. Each time a particle is picked, the time is increased by one Monte Carlo step (MCS). If we take $1 / \tau$ to be the attempt frequency, then the real time corresponding to one MCS is $\tau / N$, where $N$ is the total number of particles, assuming that each particie is excited once by the heat bath, provided by the crystal, in each time interval $\tau$. This Monte Carlo technique then is a method of "solving" the master equation

$$
\begin{aligned}
& \frac{d P\left(c_{f}, t\right)}{d t} \\
& \quad=\sum_{c_{i}} \frac{w\left(c_{i}, c_{f}\right) P\left(c_{i}, t\right)}{\tau}-\sum_{c_{i}} \frac{w\left(c_{f}, c_{i}\right) P\left(c_{f}, t\right)}{\tau},
\end{aligned}
$$

where $P(c, t)$ is the probability distribution of configurations at time $t$. For the Monte Carlo dynamics to be consistent with detailed balance at thermal equilibrium, it is necessary that

$$
w\left(c_{i}, c_{f}\right) P_{\mathrm{eq}}\left(c_{i}\right)=w\left(c_{f}, c_{i}\right) P_{\mathrm{eq}}\left(c_{f}\right)
$$

and

$$
P_{\text {eq }}(c)=Z^{-1} \exp \left(-H[c] / k_{B} T\right),
$$

where $H$ is the Hamiltonian and $Z$ is the partition function of the system, and $P_{e q}$ is the probability distribution of configurations at equilibrium. Detailed balance, of course, does not completely specify $w\left(c_{i}, c_{f}\right)$.

One possible choice for $w\left(c_{i}, c_{f}\right)$, which satisfies detailed balance, is the Metropolis walk, ${ }^{4}$ for which

$$
\begin{aligned}
w_{m}\left(c_{i}, c_{f}\right) & =\exp \left(-\delta E / k_{B} T\right) \text { for } \delta E>0 \\
& =1 \text { for } \delta E<0,
\end{aligned}
$$

where $\delta E=H\left[c_{f}\right]-H\left[c_{i}\right]$. Another common choice for $w\left(c_{i}, c_{f}\right)$, for lattice-gas systems with conservation of total number of particles is Kawasaki dynamics, ${ }^{5-7}$ for which

$$
w_{k}\left(c_{i}, c_{f}\right)=\frac{\exp \left(-\delta E / 2 k_{B} T\right)}{\exp \left(-\delta E / 2 k_{B} T\right)+\exp \left(\delta E / 2 k_{B} T\right)},
$$

and Kawasaki dynamics also satisfy detailed balance. However, the dynamics of thermally excited processes are properly described by 


$$
w_{e}\left(c_{i}, c_{f}\right)=\exp \left[-E_{b}\left(c_{i}, c_{f}\right) / k_{B} T\right],
$$

where $E_{b}\left(c_{i}, c_{f}\right)$ is the energy barrier for a particle to hop, which changes the configuration from $c_{i}$ to $c_{f}$. This has been used in some previous Monte Carlo simulations of adsorbed gases. ${ }^{8-13}$ When using Kawasaki dynamics, $\tau$ can be considered to be a temperature-dependent quantity $e^{Q / k_{B} T} / \nu$, where $Q$ is an average energy barrier that the particles have to surmount when hopping from site to site, and $v$ is the frequency of the frustrated translational motion parallel to the surface. ${ }^{1,2}$ The quantity $Q$ is temperature dependent because the configuration of the lattice gas particles is temperature dependent. However, with Kawasaki dynamics, $\tau$ is normally taken to be the unit of time, and the temperature dependence of $Q$ is lost from the simulation. From this point of view, we can consider Kawasaki dynamics to be a procedure for sampling which merely satisfies the detailed balance condition for the equilibrium configuration distribution. On the other hand, $w_{e}$ is a physical quantity: the probability of success for thermally excited barrier crossing. When using $w_{e}$ in a simulation, $\tau$ would simply be $1 / v$.

\section{RESULTS AND DISCUSSION}

The probability $w_{k}$ can be regarded as resulting from a rescaling of $w_{e}$. Consider a particle hopping from site $i$ to site $j$ over an energy barrier, as shown in Fig. 1. The relationship between $w_{k}$ and $w_{e}$ is

$$
w_{k}(i, j)=A w_{e}(i, j),
$$

where

$$
\begin{aligned}
A= & {\left[\exp \left(\frac{\delta E}{2 k_{B} T}+\frac{|\epsilon|}{k_{B} T}\right)\right] / } \\
& {\left[\exp \left(\frac{\delta E}{2 k_{B} T}\right)+\exp \left(-\frac{\delta E}{2 k_{B} T}\right)\right], }
\end{aligned}
$$

and $\epsilon$ is defined in Fig. 1. In an interacting lattice-gas system, the difference in energy for each pair of sites will depend on the local configuration around the pair. Thus, there will be a specific value of $A$ for each type of neighborhood in which a pair of sites finds itself. In the case where there is no lateral interaction between the particles of the lattice gas, there is only one value of $A$. In this case, even if the microscopic events actually occur with probabilities $w_{e}$, a simulation can be done using Kawasaki dynamics. The real time is then $t_{r}=A \tau S / N$; whereas if the simulation had been done with probabilities $w_{e}$, then the real time would be $t_{r}=\tau S / N$, where $S$ is the number of Monte Carlo steps. In order to obtain the real time, the time obtained using Kawasaki dynamics just needs to be multiplied by the factor $A$, if the underlying dynamics are actually described by the probabilities $w_{e}$. This, however, is not possible if there are lateral interactions and, hence, more than one value of $A$.

Let us now compare the processes of hopping between sites $i$ and $j$ and sites $j$ and $k$. Generally, the three sites have different energies, and the values of $A$ defined by Eq. ( 8 ) for the pairs of sites $(i, j)$ and $(j, k)$ are not equal. If we normalize $w_{e}(i, j)$ with respect to $w_{e}(i, j)+w_{e}(j, i)$, we find that

$$
\frac{w_{e}(i, j)}{w_{e}(i, j)+w_{e}(j, i)}=w_{k}(i, j) \text {. }
$$

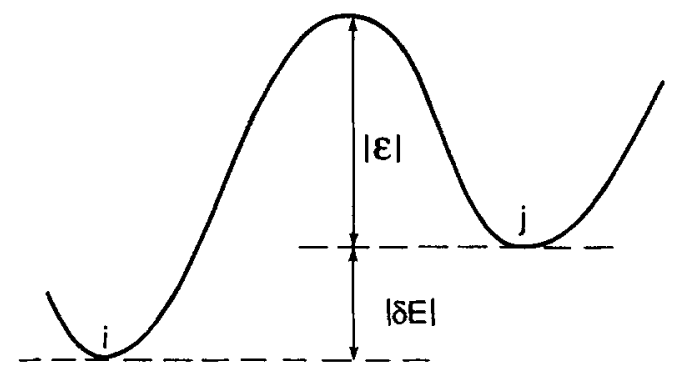

FIG. 1. Quantities used in Eq. (8) to rescale $w_{e}$ to $w_{k}$, when hopping from site $i$ to site $j$.

Hence, Kawasaki dynamics rescale the probabilities of barrier crossing so that for the barrier between any two sites $(i, j), w_{k}(i, j)+w_{k}(j, i)=1$, cf. Eq. (5). For barriers where $w_{e}(i, j)+w_{e}(j, i)>1$, the sum of the rates of hopping is decreased; whereas for barriers where $w_{e}(i, j)$ $+w_{e}(j, i)<1$, this sum is increased. As a result of this rescaling, Kawasaki dynamics require shorter computational times for simulating processes where the values of $w_{e}$ are very small. When the values of $w_{e}$ are very small, a "hop" has to be attempted many times before it is successful. Hence, computer time can become prohibitive.

In such cases, which occur when the temperature of the lattice gas is relatively low, Monte Carlo simulations should not be done by addressing particles at random. Rather, all the possible hops should be grouped according to their values of $w_{e}$. Then a particular hop (a particular particle and a particular lattice direction) is picked according to the relative probability $P_{q}=n_{q} w_{e, q} / \Sigma_{q} n_{q} w_{e, q}$ where $n_{q}$ is the number of possible hops in the $q$ th group which has a probability of success equal to $w_{e, q}$. This is done by generating a random number $r$ such that $0<r \leqslant 1$. The first value of $s$ for which $\Sigma_{q=1}^{s} P_{q} \geqslant r$ is the group of hops that is selected. One hop out of all the possible hops in the sth group is selected at random. The configuration is changed each time a particular hop is picked. This algorithm for Monte Carlo simulations, where the probability of success of a hop is determined before the hop is picked, rather than after, is called the " $n$-fold" way. ${ }^{14}$ It makes it feasible to do simulations, both dynamic and static, when the probability of a change in the configuration is much smaller than unity. In the calculation of static quantities, the configurational changes do not have to correspond to physical events. It is only important that the transition probabilities which are used produce the correct equilibrium solution of the master equation, cf. Eq. (1). However, for calculating dynamic quantities, the configurational changes correspond to real events, such as the hopping of an adatom from one adsorption site to another. Hence, for simulations of dynamic processes the choice of the time increment for each sampling step is crucial. The hop attempt frequency is $1 / \tau$, and the average number of attempts that occurs before a hop in the $q$ th group is successful is $1 / w_{e, q}$. Thus, the time is increased by $\tau / N w_{e, q}$ for each hop in the $q$ th group, where $N$ is the total number of particles. The factor of $1 / N$ occurs because each particle provides a clock, but all the $N$ clocks are running in parallel. We have used this procedure to simulate the dynamics of domain ordering on a square lattice gas 


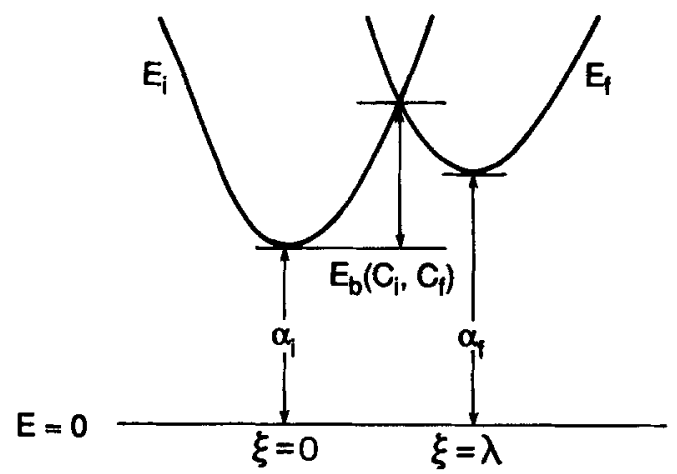

FIG. 2. Model for diffusion barrier described by Eqs. (10) and (11). The bottoms of the potential wells at the adsorption sites $i$ and $f$ are $\alpha_{i}$ and $\alpha_{f}$ respectively. The bottom of the potential well for an adsorption site with no nearest- or next-nearest-neighbor adatom is the zero of energy.

with equal and repulsive nearest- and next-nearest-neighbor interactions. At a fractional coverage of 0.5 , the lattice gas forms fourfold degenerate $(2 \times 1)$ ordered domains below a critical temperature given by $k_{B} T_{c} / \phi \sim 0.525,{ }^{15}$ where $\phi$ is the strength of the lateral interactions. The values of the barriers $E_{b}\left(c_{i}, c_{f}\right)$ are obtained from the model (harmonic) potentials shown in Fig. 2, in which

$$
E_{i}=k \xi^{2} / 2+\alpha_{i} \text {, }
$$

and

$$
E_{f}=k(\xi-\lambda)^{2} / 2+\alpha_{f},
$$

where $k$ is the force constant of the harmonic wells, $\alpha_{i}$ and $\alpha_{f}$ are the heights of the bottoms of the initial and final adsorption wells, $\zeta$ is the coordinate for hopping, and $\lambda$ is the lattice constant. In this case,

$$
\alpha_{i}=\phi N_{i},
$$

where $N_{i}$ is the total number of occupied nearest-and nextnearest-neighbor sites, i.e., the energy zero is the energy of an "isolated" adatom. The quantity $E_{b}\left(c_{i}, c_{f}\right)$ is the difference in energy between the point of intersection of $E_{i}$ and $E_{f}$ and the bottom of the well described by $E_{i}$. In our simulations, we have set $k \lambda^{2}=20 \phi,{ }^{16}$ and we have studied diffusion of the lattice gas and the kinetics of domain growth with Monte Carlo techniques using $w_{k}$ and $w_{e}$. If $\lambda \sim 3 \AA, \phi \sim 2 \mathrm{kcal} / \mathrm{mol}$ and $\mu \sim 10 \mathrm{amu}$, then $k \lambda^{2}=20 \phi$ implies an adatom frustrated translational frequency parallel to the surface of $v_{\|} \sim 72$ $\mathrm{cm}^{-1}$. We also have performed simulations with the Metropolis walk algorithm for the temperature regime where domain growth occurs.

The initial configurations are generated by randomly placing 5000 particles on a $100 \times 100$ lattice, so that the effective initial temperature for all runs is infinity and the fractional coverage is one-half. We performed 30 runs for each temperature, and obtained the mean-square displacement of the particles from the simulations for $0.6 \phi / k_{B} \leqslant T \leqslant 5 \phi / k_{B}$. After an initial period of time to allow the system to "forget" its initial configuration, the mean-square displacement varies linearly with time, with a slope proportional to the diffusion coefficient. The temperatures are sufficiently high that the effect of the order-disorder transition ${ }^{17}$ on the diffusion behavior is not observed. We plot in Fig. 3 the logarithm of

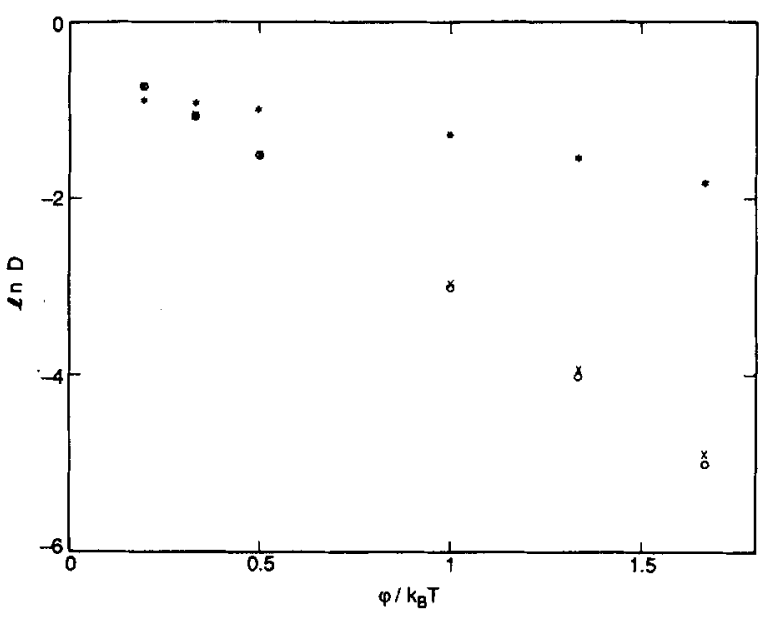

FIG. 3. Plot of $\ln D$ vs $\phi / k_{B} T$, where $D$, in units of $\lambda^{2} / \tau$, is the slope of the mean-square displacement of an adatom vs time, i.e., the diffusion coefficient. The quantity $\phi$ is the lateral interaction strength, $\lambda$ is the lattice constant, and $\tau$ is the inverse of the attempt frequency. The circles are data for simulations using $w_{e}$, the asterisks are data for simulations using $w_{k}$, and the crosses are data for simulations using $w_{k}$ but with time rescaled as explained in the text.

the slope as a function of the reciprocal of the corresponding temperature in order to obtain the effective energy barrier to diffusion. The asterisks are results from simulations using $w_{k}$, and the circles are results from simulations using $w_{e}$. Since we used only temperatures above the critical temperature, we did not observe a significant variation in the energy barrier for diffusion. The average diffusion barrier obtained using the probabilties $w_{e}$ varies from $\sim 2.6 \phi$ at the higher temperatures to $\sim 3.0 \phi$ at the lower temperatures. As the temperature is lowered the probability of finding local configurations which are energetically more favorable, and thus have a higher diffusion barrier, becomes higher. Thus, the average diffusion barrier is expected to increase slightly with decreasing temperature, even when the lattice gas is still above the critical temperature for ordering. At an infinite temperature, where the correlation length is zero, the average neighborhoods of a particle before and after a hop are the same, and $\alpha_{i}$ and $\alpha_{f}$ are equal. With our model, this gives a diffusion barrier of $2.5 \phi$.

By the same argument, at an infinite temperature, Kawasaki dynamics would show a zero energy barrier to hopping. Our results for simulations using Kawasaki dynamics, indicated by the asterisks in Fig. 3, show that the average barrier tends to zero at high temperatures. However, the values obtained from simulations using $w_{k}$ at finite temperatures cannot be easily interpreted since, as described earlier, we can consider each $w_{k}$ to result from a rescaling of a corresponding $w_{e}$. Using the procedure described earlier for small $w_{e}, t_{r}$ is increased by $\tau / N w_{e, q}$ for each hop. If a simulation is carried out with this procedure but using the probabilites $w_{k}$, the time increment for each hop is $\tau / N w_{k, q}$. If, however, for each hop in the simulations using $w_{k}$, we increase $t_{r}$ by $\tau / N A_{q} w_{k, q}$, we obtain the results shown by the crosses in Fig. 3. The results are also shown in Table I. The additional factor of $A_{q}$ for each hop, the value of $A_{q}$ depending on the type of hop, means that the correct time increment 
is used for each hop. As discussed earlier the probabilities $w_{k}$ are obtained by rescaling the values of $w_{e}(i, j)$ and $w_{e}(j, i)$ by $w_{e}(i, j)+w_{e}(j, i)$. For temperatures which are relatively low, each value of $w_{e}$ is very small, and this rescaling increases the probability of hopping so that the diffusion coefficient is higher when using Kawasaki dynamics. However, for temperatures which are sufficiently high so that $w_{e}(i, j)+w_{e}(j, i)>1$, this rescaling would actually lower the probability of hopping and, hence, lower the diffusion coefficient. This may be seen by comparing the asterisks and the crosses in Fig. 3. The rescaled Kawasaki results are in good agreement with those using the proper energy barrier at temperatures which are high compared to $\phi / k_{B}$, with progressively greater disagreement at progressively lower tempeatures. This may be seen by comparing the circles and the crosses in Fig. 3, and it is shown more clearly in Fig. 4 where we plot the temperature dependence of $D_{r} / D_{e}$, the ratio of the diffusion coefficient obtained from using $w_{k}$ (and rescaling the time for each hop by $A_{q}$ ) to the diffusion barrier obtained from using $w_{e}$. This behavior is observed because at high temperatures the relative probabilities $P_{q}$ calculated from $w_{e}$ and from $w_{k}$ for each type of hop are approximately the same. However, the lower the temperature the greater the difference between the value of $P_{q}$ calculated from $w_{e}$ and that calculated from $w_{k}$. This affects the time evolution since the moves are picked according to their relative probabilities. Hence, as discussed earlier, in lattice-gas systems with lateral interactions, simulations using Kawasaki $d y$ namics are not equivalent to simulations using $w_{e}$. Only when there are no lateral interactions can rescaling the time give equivalent results. At sufficiently high temperatures, using Kawasaki dynamics is approximately equivalent to using $w_{e}$ because $A_{q} \sim 1 / 2$, a constant regardless of the local configuration. Simulations using the Metropolis walk would be expected to also show similar deviations from simulations using $w_{e}$.

Lattice-gas diffusion has been investigated rather extensively by Monte Carlo methods. In some of these studies, the transition probabilities were computed by considering the energy difference between the final and initial configurations. Both Kawasaki dynamics ${ }^{17-19}$ and the Metropolis walk $^{20,21}$ have been used. In other studies ${ }^{8-13}$ the transition probabilities were computed by considering the energy barrier between the final and the initial configurations. We have shown here that the particular choice of transition probabilities influences the temperature dependence of the diffusion coefficient. As was noted previously in studies where Metropolis walk simulations were used to obtain the temperature dependence of the diffusion coefficient, these time-dependent Monte Carlo simulations used a clock, the time scale of which is not known. ${ }^{21}$ If another set of transition probabilities such as $w_{e}$ or $w_{k}$ were used, then the ratio of the calculated diffusion coefficients at a specific temperature would depend on the ratio of the respective time scales. This would not be an obstacle if the ratio were independent of the temperature. However, we have shown that this is not the case, and that the experimentally accessible temperature dependence of the diffusion coefficient for thermally excited motion of adsorbed particles can be incorrectly computed if inappropriate transition probabilities are used.

Kawasaki dynamics ${ }^{5-7}$ have been widely used to study the kinetics of domain growth. ${ }^{22-34}$ One of the major issues is the value of the growth exponent $x$ defined by $l \sim t^{x}$, where $l$ is an average length scale of the ordered domains and $t$ is the time. Since such simulations are for temperatures that are low compared to $\phi / k_{B}$, it is very important to compare the results from simulations using $w_{k}$ and $w_{e}$. The results from simulations of lattice-gas diffusion above have shown that the ratio of the time scales for simulations using $w_{e}$ and $w_{k}$ is temperature dependent, i.e., we have shown that there is a temperature-dependent multiplicative factor relating the time scales in simulations using $w_{e}$ and simulations using $w_{k}$. This multiplicative difference between the time scales would not affect the isothermal value of the growth exponent. However, it is not clear whether a linear relationship holds between the time scales of Monte Carlo simulations which use different values of $w\left(c_{i}, \mathcal{c}_{f}\right)$. Under conditions where the simulations have reached equilibrium, this is not an important consideration. In this case, the average value of the factor $A$, cf. Eq. (8), is constant since the distribution of types of hops that are taken is stationary. Hence, a linear relationship holds between the time scales of simulations using $w_{e}$ and those using $w_{k}$ or $w_{m}$. This is the reason for observing, in the diffusion simulations discussed above, the same linear scaling of mean-square displacement with time whether $w_{e}$ or $w_{k}$ was used. However, for simulations where the system is far from equilibrium, the distribution of types of hops that are taken changes as the simulations proceed. This is the case for simulations of domain growth. Since $x$ is the exponent of the time, the value of $x$ obtained from simulations can be accurate only if $x$ is obtained in a regime of domain growth where the distribution of types of hops that are taken in the simulations changes in concert with that in a real system. We cannot compare simulations directly with a real system, but we can compare the time evolution in simulations that use different transition probabilites. This would

TABLE 1. The diffusion coefficients are in units of $\lambda^{2} \tau^{-1}$. The subscripts $e, r$, and $k$ denote energy barrier, rescaled Kawasaki, and Kawasaki, respectively. The standard deviations are shown for each datum point.

\begin{tabular}{cccc}
\hline$k_{B} T / \varphi$ & $D_{e}$ & $D_{r}$ & $D_{k}$ \\
\hline 0.60 & $0.00675 \pm 0.00007$ & $0.00751 \pm 0.00009$ & $0.159 \pm 0.017$ \\
0.75 & $0.0178 \pm 0.0002$ & $0.0196 \pm 0.0003$ & $0.213 \pm 0.003$ \\
1.00 & $0.0486 \pm 0.0006$ & $0.00512 \pm 0.0005$ & $0.273 \pm 0.003$ \\
2.00 & $0.219 \pm 0.003$ & $0.218 \pm 0.002$ & $0.367 \pm 0.004$ \\
3.00 & $0.343 \pm 0.004$ & $0.347 \pm 0.004$ & $0.395 \pm 0.006$ \\
5.00 & $0.479 \pm 0.004$ & $0.484 \pm 0.005$ & $0.403 \pm 0.003$ \\
\hline
\end{tabular}




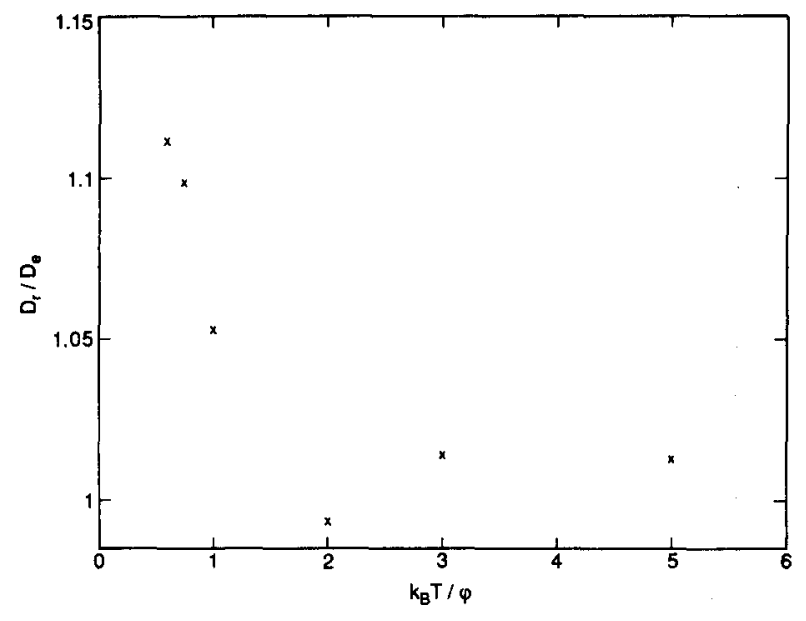

FIG. 4. Plot of $D_{r} / D_{e}$ vs $k_{B} T / \phi$. The quantity $D$, is the diffusion coefficient obtained for simulations using $w_{k}$ and rescaled time. The quantity $D_{e}$ is the diffusion coefficient obtained for simulations using $w_{e}$.

determine whether or not the choice of the transition probabilities is important for simulations of systems evolving towards equilibrium.

We have performed simulations at a temperature $T=0.3325 \phi / k_{B}$ using $w_{k}, w_{m}$, and $w_{e}$. For the simulations for $w_{e}$ and $w_{m}$, we used a $L \times L=100 \times 100$ lattice and performed 40 runs. For the simulations for $w_{k}$, we performed only 15 runs since reliable data are already available for this case. ${ }^{31}$ All simulations were allowed to run sufficiently long that the final domain sizes are approximately $L / 3$. For the simulations using $w_{e}$ and $w_{m}$, we also performed five runs each on $200 \times 200$ lattices to ensure that "finite-size effects" do not influence the results. Time is measured in Monte Carlo steps, as usual, for the simulations using both Kawasaki dynamics and the Metropolis walk. For the simulations using $w_{e}$, real time is calculated explicitly, as discussed earlier. The results are shown in Figs. 5-8. Simulations using

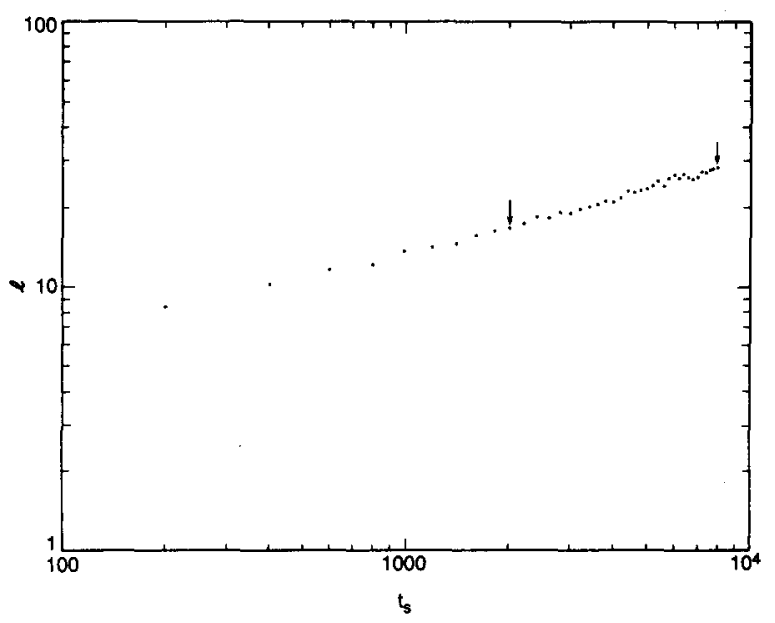

FIG. 5. Average of 15 Kawasaki dynamics runs for ordering of fourfold degenerate $(2 \times 1)$ domains on a square lattice at a fractional coverage of one-half and a temperature of $0.3325 \phi / k_{B}$. The quantity $\phi$ is the lateral interaction strength. The lattice size used is $100 \times 100$. The best fit for the growth exponent using the data points between the arrows is $x_{k}=0.34 \pm 0.01$. This agrees well with the extensive work of Sadiq and Binder who obtained a value of $x_{k} \sim 0.35$ (Ref. 30).

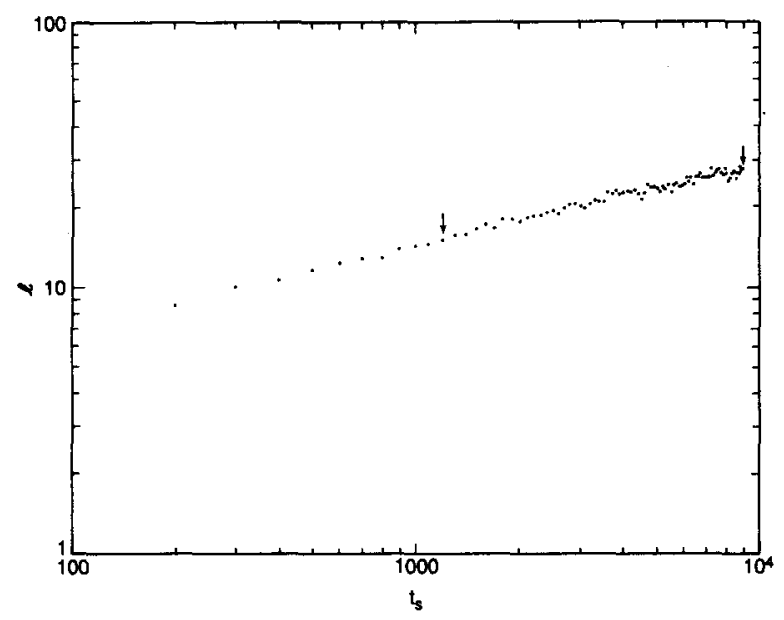

FIG. 6. Average of 45 Metropolis runs for the same system as in Fig. 5 . Forty runs were done on a lattice of $100 \times 100$, and five runs were done on a lattice of $200 \times 200$. For the domain sizes that were reached, no finite-size effects were observed. The best fit for the growth exponent using the data points between the arrows is $x_{m}=0.277 \pm 0.007$. If all the data points are used, a best fit of $x_{m}=0.300 \pm 0.008$ is obtained.

Kawasaki dynamics for this system at the same temperature have been performed previously, and our results for Kawasaki dynamics, which are shown in Fig. 5, agree with those simulations. ${ }^{31}$ The growth exponent we obtained using Kawasaki dynamics is $x_{k} \sim 0.34 \pm 0.01$, where the uncertainty here (and below) refers to one standard deviation in a linear least-squares fit to the calculated data. This growth exponent is obtained from the data points between the arrows indicated in Fig. 5. In Fig. 6 we show the results from simulations using the Metropolis walk, where the data points between the arrows give a growth exponent of $x_{m} \sim 0.277 \pm 0.007$. (If all data points are included, the best fit gives $x_{m} \sim 0.300 \pm 0.008$.) Figure 7 shows the results from a single run using $w_{e}$, and Fig. 8 shows the average of 45 runs using $w_{e}$. The data points between the arrows in Fig. 8 give $x_{e} \sim 0.329 \pm 0.004$. Just as we can regard $w_{k}\left(c_{i}, c_{f}\right)$ to be the result of rescaling $w_{e}\left(c_{i}, c_{f}\right)$ with respect to $w_{e}\left(c_{i}, c_{f}\right)$

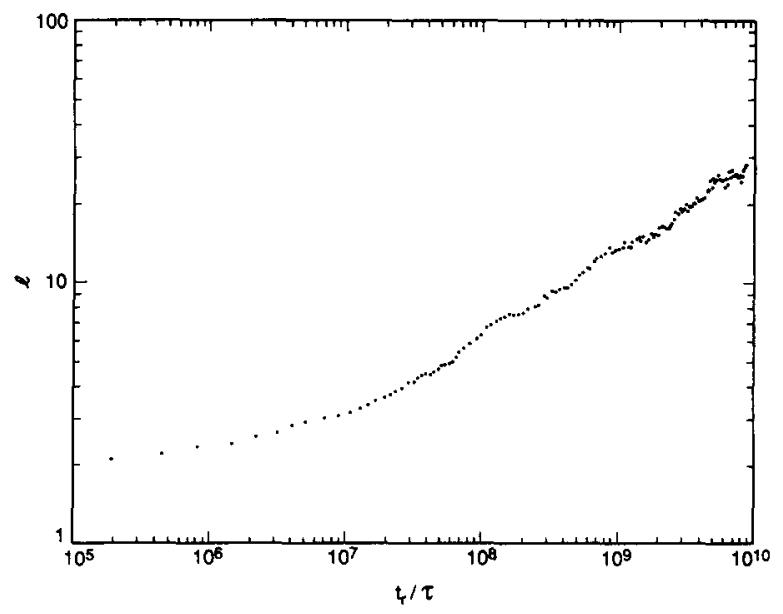

FIG. 7. A typical single run using the energy-barrier model. The fractional coverage is one-half and the temperature is $0.3325 \phi / k_{B}$, i.e., the same as in Figs. 5 and 6. The additional parameter necessary in these simulations is the force constant of the model harmonic potential wells illustrated in Fig. 2. For our simulations, we have set the force constant to be $k=20 \phi / \lambda^{2}$, where $\phi$ is the lateral interaction strength and $\lambda$ is the lattice constant. 


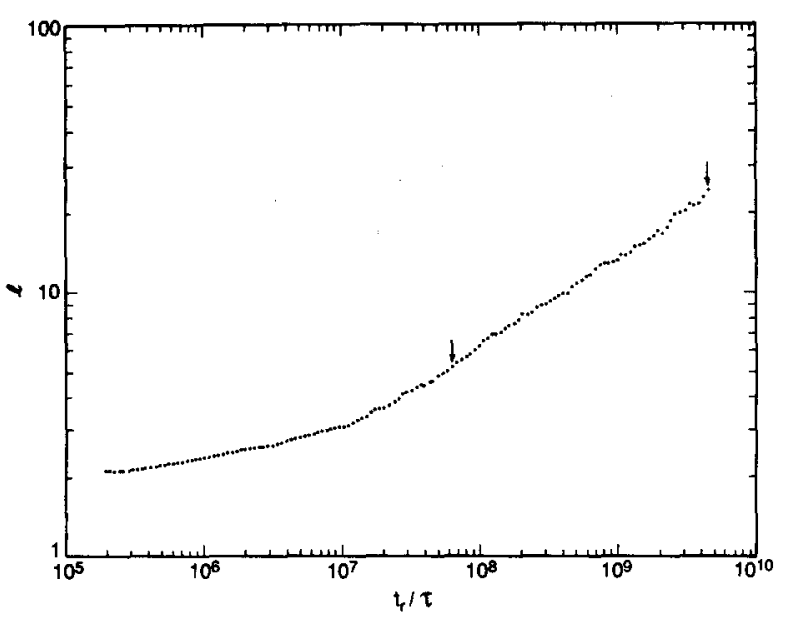

FIG. 8. Average of 45 runs using the energy-barrier model, of which a typical single run is shown in Fig. 7. (The simulation parameters are summarized there.) Forty runs were done on a lattice of $100 \times 100$, and five runs were done on a lattice of $200 \times 200$. For the domain sizes reached here, no finite-size effects were observed. The best fit for the growth exponent using the data points between the arrows is $x_{e}=0.329 \pm 0.004$.

$+w_{e}\left(c_{i}, c_{f}\right)$, we can consider $w_{m}\left(c_{i}, c_{f}\right)$, to be the result of rescaling $w_{e}\left(c_{i}, c_{f}\right)$, with respect to $\max \left[w_{e}\left(c_{i}, c_{f}\right)\right.$, $\left.w_{e}\left(c_{f}, c_{i}\right)\right]$. Our results show that $t_{s}$ in the simulations using the Metropolis algorithm does not scale linearly with $t_{s}$ in the simulations using Kawasaki dynamics. The values of $x_{k}$ and $x_{e}$, however, are in rather close agreement. This means the value of $x$ can be affected by the type of transition probabilities that is used even though the total particle number is conserved in all cases. This, as we suggested above, can arise because the distribution of hops that are taken is changing in these simulations, and they do not change in concert for simulations using different forms for $w\left(c_{i}, c_{f}\right)$.

We conclude that all interpretations of dynamic Monte Carlo simulations must be made with some care. For instance, the value of $x=1 / 3$ has been suggested for the system that we have studied on the basis of simulations using Kawasaki dynamics. ${ }^{34}$ It was argued that this value results from the long-range diffusion of lattice-gas particles from domain walls with excess local density to domain walls with deficit local density. Clearly, the same long-range diffusion of lattice-gas particles must also occur when a Metropolis walk is used. However, our results indicate a significantly different growth exponent for the Metropolis walk. We can conclude that with other constraints the same (such as the conservation law for the total particle number and the degeneracy of the ground state), the choice of the transition probabilities can influence the value of $x$ that is calculated. This can be interpreted to mean that if the microscopic processes, in two otherwise similar systems, have different forms for $w\left(c_{i}, c_{f}\right)$, then the values of $x$ could be different. This serves to underscore in a dramatic fashion the importance of correctly converting simulation time to real time, and this occurs naturally for thermally excited processes when employing the proper energy-barrier model that we have developed.

\section{CONCLUSIONS}

In conclusion, we have demonstrated the importance of the choice of the transition probabilities in Monte Carlo sim- ulations. We find that the ratio of time scales using different choices for $w\left(c_{i}, c_{f}\right)$ is dependent on temperature and affects calculations of diffusion coefficients, particularly at low temperatures. We also find that under nonequilibrium conditions the time scales using different choices for $w\left(c_{i}, c_{f}\right)$ do not necessarily scale linearly. This affects the calculation of dynamic quantities such as the growth exponent of ordered domains using Monte Carlo simulations. In general, the correct growth exponent can be obtained only if the transition probabilities that are used correctly describe the microscopic dynamics, which for thermally excited processes have the form $e^{-E_{b}\left(c_{b} c_{f}\right) / k_{B} T}$. Specifically, we find that for the ordering of fourfold degenerate $(2 \times 1)$ domains on a square lattice,the growth exponent obtained using the Metropolis walk is $x_{m} \sim 0.277$, and is significantly different from the value $x_{e} \sim 0.329$ obtained from simulations using $w_{e}$ within the context of an energy-barrier crossing model we have developed. The value of $x_{e}$ is quite close to that of $x_{k}$ (using Kawasaki dynamics) so that the nonlinearity between the timescales using $w_{e}$ and $w_{k}$ is not large, at least in the regime where the domain growth exponents were obtained. However, as we have argued above, $x_{e}$ not $x_{k}$ should be the correct growth exponent for the thermally excited process of domain growth of adsorbed particles.

\section{ACKNOWLEDGMENT}

This research was supported by the National Science Foundation under Grant No. DMR-8500789.

\footnotetext{
${ }^{1}$ Monte-Carlo Methods in Statistical Physics, Vol. 7 of Topics in Current Physics, edited by K. Binder (Springer, Heidelberg, 1979).

${ }^{2}$ Applications of the Monte-Carlo Methods in Statistical Physics, Vol. 36 of Topics in Current Physics, edited by K. Binder (Springer, Heidelberg, 1983).

${ }^{3}$ O. G. Mouritsen, Computer Studies of Phase Transitions and Critical Phenomena (Springer, Heidelberg, 1984).

${ }^{4}$ M. Metropolis, A. W. Rosenbluth, M. N. Rosenbluth, A. N. Teller, and E. Teller, J. Chem. Phys. 21, 1087 (1953).

${ }^{5}$ K. Kawasaki, Phys. Rev. 145, 224 (1966).

${ }^{6}$ K. Kawasaki, Phys. Rev. 148, 375 (1966).

${ }^{7}$ K. Kawasaki, Phys. Rev. 150, 285 (1966).

${ }^{8}$ M. Bowker and D. A. King, Surf. Sci. 71, 583 (1978).

${ }^{9}$ D. A. Reed and G. Ehrlich, Surf. Sci. 102, 588 (1981).

${ }^{10}$ D. A. Reed and G. Ehrlich, Surf. Sci. 105, 603 (1981).

${ }^{1}$ 'E. S. Hood, B. H. Toby, and W. H. Weinberg, Phys. Rev. Lett. 55, 2437 (1985).

${ }^{12}$ C. H. Mak, H. C. Andersen, and S. M. George, J. Chem. Phys. 88, 4052 (1988).

${ }^{13}$ H. C. Kang and W. H. Weinberg (unpublished).

${ }^{14}$ A. B. Bortz, M. H. Kalos, and J. L. Lebowitz, J. Comp. Phys. 17, 10 (1975).

${ }^{15}$ K. Binder and D. P. Landau, Phys. Rev. B 21, 1941 (1980).

${ }^{16}$ Additional runs with $k \lambda^{2}=800 \phi$ show that the growth exponent is not influenced to within statistical error by a change in the value of $k \lambda^{2} / \phi$.

${ }^{17}$ A. Sadiq and K. Binder, Surf. Sci. 128, 350 (1983).

${ }^{18}$ R. Kutner, K. Binder, and K. Kehr, Phys. Rev. B 26, 2967 (1982).

${ }^{19}$ R. Kutner, K. Binder, and K. Kehr, Phys. Rev. B 28, 1846 (1983).

${ }^{20} \mathrm{~N}$. Tringides and R. Gomer, Surf. Sci. 145, 121 (1984).

${ }^{21}$ X. P. Jiang and H. Metiu, J. Chem. Phys. 88,1891 (1984).

${ }^{22}$ K. Binder and D. Stauffer, Phys. Rev. Lett. 33, 1006 (1974).

${ }^{23}$ K. Binder, C. Billotet, and P. Mirold, Z. Phys. B 30, 183 (1978).
} 
${ }^{24}$ K. Binder, Phys. Rev. B 15, 4425 (1977).

${ }^{25}$ J. Marro, J. L. Lebowitz, and M. H. Kalos, Phys. Rev. Lett. 30, 289 (1982).

${ }^{26}$ P. S. Sahni, J. D. Gunton, S. L. Katz, and R. H. Timpe, Phys. Rev. B 25, 289 (1982).

${ }^{27}$ P. S. Sahni and J. D. Gunton, Phys. Rev. Lett. 45, 369 (1980).

${ }^{28}$ S. A. Safran, Phys. Rev. Lett. 46, 1581 (1981).
${ }^{29}$ P. S. Sahni and J. D. Gunton, Phys. Rev. Lett. 47, 1754 (1981).

${ }^{30}$ P. S. Sahni, G. Dee, J. D. Gunton, J. K. Phani, J. L. Lebowitz, and M. H. Kalos, Phys. Rev. B 24, 410 (1981).

${ }^{31}$ S. Sadiq and K. Binder, J. Stat. Phys. 35, 517 (1984).

${ }^{32}$ P. S. Sahni, G. S. Grest, and S. A. Safran, Phys. Rev. Lett. 50, 60 (1983).

${ }^{33}$ S. A. Safran, P. S. Sahni, and G. S. Grest, Phys. Rev. B 28, 2693 (1983).

${ }^{34} \mathrm{~J}$. Vinals and J. D. Gunton, Surf. Sci. 157, 473 (1985). 